TËOROS $\begin{aligned} & \text { Téoros } \\ & \text { Revue de recherche en tourisme }\end{aligned}$

37,2 | 2018

Sexualités touristiques

\title{
Investissements par amour. Économies intimes de l'immobilier touristique au Brésil
}

\author{
Tristan Loloum
}

\section{(2) OpenEdition}

Journals

Édition électronique

URL : http://journals.openedition.org/teoros/3337

ISBN : 1923-2705

ISSN : 1923-2705

Éditeur

Presses de l'Université du Québec

Ce document vous est offert par Université de Tours

\section{université
de TOURS}

Référence électronique

Tristan Loloum, «Investissements par amour. Économies intimes de l'immobilier touristique au Brésil », Téoros [En ligne], 37, 2 | 2018, mis en ligne le 20 mai 2018, consulté le 11 juin 2018. URL : http:// journals.openedition.org/teoros/3337

Ce document a été généré automatiquement le 11 juin 2018.

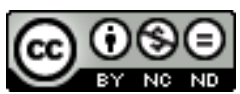

La revue Téoros est mise à disposition selon les termes de la Licence Creative Commons Attribution Pas d'Utilisation Commerciale - Pas de Modification 4.0 International. 


\title{
Investissements par amour. Économies intimes de l'immobilier touristique au Brésil
}

\author{
Tristan Loloum
}

1 En 2010, alors que j'effectuais une enquête sur le développement des résidences secondaires dans le Nordeste, un promoteur catalan installé à Ponta Negra, dans l'État du Rio Grande do Norte (RN), me confiait que « $90 \%$ de [ses] clients étaient des hommes qui cherchaient un logement pour leur petite copine. Certains tombaient amoureux d'une prostituée ou d'une femme d'ici et s'achetaient un appartement. [Il] a vendu beaucoup d'appartements grâce aux prostituées... " Le fait que les rencontres amoureuses et la prostitution puissent être des moteurs du boom immobilier me parut d'emblée constituer un intéressant sujet d'anthropologie économique.

2 Nous étions alors au lendemain de la crise économique initiée en 2008 et nombre des projets immobiliers destinés à une clientèle européenne étaient désormais à l'arrêt. Parmi les économistes, les théories hétérodoxes suscitaient un regain d'intérêt, notamment du côté des comportementalistes, dont certains évoquaient le rôle des "esprits animaux " des agents économiques dans les krachs financiers (Akerlof et Schiller, 2009). Il est vrai que l'épisode spéculatif précédant l'effondrement des marchés immobiliers avait quelque chose d'exubérant (Loloum et Aledo, à paraître 2018), et certains mégaprojets immobiliers, manifestement surdimensionnés par rapport à la demande et aux infrastructures existantes, paraissaient " gonflés à la testostérone » (Cueva et al., 2015).

D'autres entretiens menés à Praia da Pipa ${ }^{1}$ - une destination touristique voisine - auprès d'acquéreurs de résidences secondaires et d'entrepreneurs étrangers confirmaient le rôle essentiel que pouvaient jouer les relations intimes dans les décisions d'achat immobilier, sans d'ailleurs que cela ait un lien avec la prostitution évoquée par mon interlocuteur de Ponta Negra ${ }^{2}$ : telle personne, après être tombée en amour avec une ou un local, avait investi dans l'achat de terrains ; tel couple s'était constitué ou au contraire défait pendant la construction de leur auberge; tel autre couple s'était rencontré à l'étranger et envisageait l'achat d'un bien immobilier comme un moyen de s'installer au Brésil, etc. 
Dans les registres fonciers de Praia da Pipa, il était par ailleurs monnaie courante de voir figurer des couples mixtes sur les contrats de propriété.

Autant d'observations qui posaient la question de la place de l'intime dans le marché de l'immobilier de loisirs, et vice versa, le rôle des achats immobiliers dans la constitution d'un couple, a fortiori lorsqu'il s'agit d'un couple mixte. Que se passe-t-il lorsqu'un couple mixte décide d'acquérir une résidence - principale ou secondaire - dans une destination touristique ? En quoi la mixité conjugale influe-t-elle sur l'acte d'achat? Plutôt que de m'intéresser à la rencontre, je m'intéresse donc dans cet article aux investissements immobiliers comme moments critiques de la vie de couple, révélateurs des coûts mais aussi de l'expatriation amoureuse et de ses rétributions (affectives, professionnelles, financières). En quoi le caractère touristique du lieu agit-il sur le couple et sa décision ? Si le sentiment d'évasion (Réau, 2005) propre à l'expérience touristique est propice à tous les excès et les rêves, l'achat d'un appartement ou d'une maison - en couple et dans un contexte international - constitue bien souvent un rappel à la réalité dès lors que se posent des questions très concrètes quant à la pérennité de l'investissement, mais aussi du couple lui-même.

\section{Contraintes et rétributions de l'expatriation amoureuse}

Ces questions font écho à une littérature scientifique située au carrefour des études du tourisme, du genre et des migrations. Bien que de nombreux travaux portent sur les espaces touristiques en tant qu'espaces de rencontre sexuelle et conjugale, à travers notamment la thématique du «tourisme sexuel» (Herold et al., 2001; Roux, 2011; Bottero, 2015), plus rares sont ceux qui s'intéressent au devenir de ces relations au-delà de la parenthèse des vacances (Despres, 2017); à moins de faire référence à une littérature sur les couples binationaux (Alber et al., 2012) et sur la globalisation des relations amoureuses (Constable, 2003), où la dimension touristique n'est souvent traitée que de façon périphérique.

Les représentations contemporaines des couples binationaux impliquant un Brésilien ou une Brésilienne sont souvent très connotées. Elles mettent généralement en scène de riches Occidentaux usant d'un statut économique dominant pour séduire de jeunes Brésiliens, eux-mêmes intéressées et usant de leurs charmes pour s'enrichir auprès de "gringos" fortunés et un tantinet naïfs. Si ces stéréotypes concernent surtout des hommes occidentaux face à des femmes brésiliennes, il n'est pas rare d'observer des situations impliquant des femmes occidentales et des hommes brésiliens, évoquées à travers la problématique des caça-gringas (Cantalice, 2016), équivalent brésilien des beach boys (Herold et al., 2001; Kempadoo, 2001). Ces rencontres suscitent toujours un certain nombre de sous-entendus quant au caractère asymétrique et instrumental de la relation. À rebours des idées préconçues, des anthropologues se sont attachés à montrer la complexité des itinéraires personnels et des «idiomes relationnels» (Simoni, 2016: 14-16) déployés à travers les rapports amoureux transnationaux.

7 Portant sur des cas d'étude brésiliens, les travaux de chercheurs comme Jordi Roca Girona, Monserrat Masdeu et Yolanda Puerta (2012) et Adriana Piscitelli (2012) remettent en cause bon nombre d'opinions communes quant à l'asymétrie des relations amoureuses Nord-Sud. À l'encontre de l'image de la femme brésilienne n'ayant que son pouvoir de séduction pour s'en sortir, leurs recherches montrent au contraire les multiples ressources (culturelles, sociales et financières) sur lesquelles peuvent compter ces 
femmes lorsqu'elles entreprennent leur "migration par amour» ou «importent» un mari/compagnon étranger. Il semblerait que les femmes brésiliennes migrant par amour ou importatrices d'amour soient même bien souvent plus instruites et issues de milieux socialement plus favorisés que les Européens « importateurs » d'amour. De même, au-delà de l'image du riche Européen venant «faire son marché » matrimonial ou sexuel au Brésil, leurs observations soulignent qu'il s'agit aussi parfois d'individus déclassés sur le marché sexuel et matrimonial européen, cherchant des solutions de "reclassement " grâce à l'expatriation. Leurs travaux remettent également en question les bénéfices attachés à la migration amoureuse des femmes brésiliennes. Perçue comme une chance d'ascension sociale, l'expatriation cache bien souvent des situations de vulnérabilité, où le conjoint expatrié se retrouve dans une situation de dépendance (économique, sociale, linguistique) à l'égard de son compagnon importateur d'amour et de son entourage. Les travaux d'Adriana Piscitelli (2016) sur les femmes européennes installées dans le Nordeste brésilien dévoilent également le décalage entre l'image fantasmée de l'homme " autochtone» [nativo] - le «beau surfeur», "l'authentique pêcheur»- et la réalité parfois violente de la relation, faite de conceptions divergentes du genre et d'antagonismes de classe entre des femmes européennes issues de classes moyenne et aisée et des «locaux» issus de milieux plus modestes, peu instruits et imprégnés d'un machisme à fleur de peau.

8 L'enquête d'Altaïr Despres (2017) sur l'expatriation amoureuse de femmes occidentales à Zanzibar insiste au contraire sur les rétributions affectives et professionnelles de ces mobilités. Elle montre en effet que l'engagement dans une relation durable avec des Tanzaniens a permis aux femmes interrogées de redonner un second souffle à leur vie affective, mais aussi à leur carrière professionnelle dès lors que le Zanzibar offre de nombreuses possibilités d'emploi aux personnes expatriées, généralement plus qualifiées que les travailleurs locaux. Elle insiste également sur le fait que la réussite de l'expatriation est autant déterminée par les disponibilités que par les dispositions au départ des femmes en question: concrètement, la migration "par amour " a d'autant plus de chances de réussir que leur vie amoureuse et professionnelle en Occident se trouvait au point mort (autrement dit que rien ou presque ne les retenait dans leur pays) et qu'elles disposaient d'un capital de sécurité pour assurer une reconversion dans le pays d'accueil. Despres montre enfin que la " dépendance » (linguistique, culturelle, sociale) de l'expatriée vis-à-vis du conjoint tanzanien peut aussi être interprétée comme une association complémentaire avec le conjoint, permettant à l'expatriée d'accéder à un certain nombre de ressources locales auxquelles elle n'aurait jamais accès en temps normal, en tant qu'étrangère, par exemple pour acquérir des terres ou pour se frayer un chemin dans les méandres de l'Administration.

9 Ces différents exemples ont pour intérêt de montrer la variabilité des configurations conjugales internationales ainsi que l'importance de prendre en compte les dispositions objectives de chacun des partenaires face à l'épreuve de l'expatriation, tant au départ qu'à l'arrivée. Au-delà des idées reçues sur les asymétries du couple mixte, ils soulignent aussi l'ambiguïté de la mixité conjugale, qui peut être aussi bien une contrainte qu'une ressource. 


\section{«Économies intimes » de l'immobilier touristique}

10 L'analyse de petits investissements immobiliers internationaux réalisés à cette époque révèle la forte implication des proches (amis, famille) dans les opérations. Ceux-ci sont en effet régulièrement appelés à prendre part au capital de la société, à acheter ou louer un appartement. Ces réseaux familiaux et amicaux sont d'autant plus importants pour valoriser un projet que les incertitudes du marché immobilier-touristique sont grandes. Mais tout en compensant la difficile lisibilité du marché, la confusion entre relations commerciales, familiales et amicales ajoute un degré de complexité supplémentaire à la gestion de l'investissement. À cette complexité affective des projets immobilierstouristiques, faite de conceptions multiples du couple, de la famille, de l'amitié et du genre entre étrangers/étrangères et nationaux/nationales, s'ajoutait un climat économique singulier marqué par l'optimisme exacerbé des années pré-2008 et l'imaginaire utopique - et parfois trompeur- des destinations tropicales dites " paradisiaques ». Dans le Nordeste, la bulle immobilière des années 2000 se manifeste par une multiplication de «resorts» et de «condominiums» (résidences sécurisées) à destination d'une demande touristique européenne jugée intarissable. En tant que marché «émergeant», le Nordeste se caractérise alors par un fort potentiel de croissance, mais aussi une grande instabilité des institutions et des infrastructures locales. Tout projet immobilier est immanquablement soumis à un éventail d'aléas bureaucratiques et politiques. Pour les promoteurs et investisseurs étrangers, cette incertitude est double: d'une part car ils maîtrisent mal les codes légaux, culturels et linguistiques de la région, et d'autre part car ils font souvent l'objet d'un traitement particulier (défavorable) de la part des acteurs locaux. Dans ce contexte d'« information imparfaite ", la relation amoureuse ou amicale avec un ou une local(e) peut apparaître comme une ressource pour contrecarrer les incertitudes de marché et les barrières à l'entrée.

11 L'enquête ethnographique invite à saisir l'épaisseur sociale et culturelle des investissements immobiliers, en dépassant ce que Viviana Zelizer (2005) appelle la doctrine des "sphères séparées" (ou des «mondes hostiles») qui tend à renvoyer l'affectif et les solidarités familiales au domaine du domestique (gemeinschaft), et les décisions rationnelles et autres calculs d'efficacité au domaine du marché et de l'individu (gesellschaft). Entreprise et famille ne sont pas deux domaines cloisonnés (Bessiere, 2010). L'anthropologie économique s'appuie ainsi sur une définition élargie de la notion d' oikonomia, entendue comme «l'art de gouverner la maison » (L'Estoile, 2014), invitant à dépasser les dichotomies d'usage qui empêchent de comprendre les pratiques économiques encastrées dans les décisions d'ordre conjugal et familial. Le concept d'« économie intime» (Zelizer, 2007; Roux, 2011) est une manière positive de qualifier cette imbrication, sans passer par des catégories du manque consistant à décrire un phénomène par ce qu'il n'est pas (par exemple le terme d'« économie informelle ») et qui renvoie toujours à l'horizon normatif d'un modèle perçu comme universel (l'« économie formelle »). S'intéresser aux « investissements par amour » et aux « économies intimes » de l'immobilier touristique, c'est dès lors étudier comment les affects, les relations personnelles et les conceptions culturelles de l'amour informent les décisions économiques, mais aussi comment ces investissements peuvent transformer le cours d'une vie et d'une relation. 


\section{Un « boom » immobilier-touristique international} internationaux sous la forme de résidences secondaires, de complexes hôteliers et résidentiels. L'euphorie immobilière gagne le littoral à partir de 2002 et s'accompagne d'un accroissement rapide de la fréquentation de touristes nationaux et internationaux. Entre 2002 et 2005, le nombre de vols hebdomadaires directs entre des villes européennes et Natal passe de 5 à 18 , ce qui a pour effet de diminuer le prix des billets. Selon INFRAERO, l'agence brésilienne des infrastructures aéroportuaires, l'État du Rio Grande do Norte aurait reçu en 2006 un total de 2186880 visiteurs, dont $30 \%$ d'étrangers. Les touristes européens jouissent alors d'un taux de change avantageux : entre 2003 et 2005, un euro s'échange pour près de 4 réaux brésiliens $(\mathrm{R} \$)$. Dans l'État du Rio Grande do Norte, $90 \%$ des investissements directs étrangers réalisés entre 2001 et 2006 sont d'origine européenne, dont $56 \%$ portugais et $14 \%$ espagnols (Ferreira, Silva et Fonseca, 2009: 129). Parmi ces capitaux, la part dédiée au secteur immobilier et touristique augmente de façon exponentielle jusqu'à la crise économique internationale de 2008, qui affectera durablement la clientèle touristique européenne. Une enquête réalisée en 2007 pour le ministère du Tourisme brésilien (Fundação Getúlio Vargas et al., 2008 : 26) montre que dans ce secteur de marché, $30 \%$ des ressources financières allouées à l'achat de résidences secondaires sont le fait de sociétés étrangères, $6 \%$ de sociétés brésiliennes, $42 \%$ proviennent de fonds de particuliers et $22 \%$ de prêts bancaires. L'étude révèle par ailleurs qu'il s'agit d'une clientèle plutôt âgée, masculine et relativement instruite : 66 \% des résidents secondaires étrangers ont entre 40 et 69 ans, $72 \%$ sont des hommes et $65 \%$ ont un diplôme d'enseignement supérieur (ibid. : 20).

Schématiquement, on peut distinguer trois phases de développement immobilier : 1) une première phase de petits investissements pionniers (2000-2005); 2) une phase de consolidation marquée par l'arrivée de grands groupes internationaux (2006-2008) ; 3) un « atterrissage en douceur » après la crise économique de 2008, compensée par le maintien d'une demande domestique jusqu'au ralentissement de l'économie brésilienne en 2012.

mière phase (2000-2005) est caractérisée par des "stratégies opportunistes 》 (David, 2012) et un secteur immobilier-touristique fragmenté, composé d'investisseurs pionniers et d'entrepreneurs isolés, disposés à prendre des risques face à un marché mal sécurisé, où les pourvoyeurs professionnels manquent. Le foncier côtier est particulièrement fragmenté, ce qui contraint les investisseurs à passer beaucoup de temps à négocier avec de petits propriétaires, à vérifier les droits de propriété et à régulariser les titres auprès des autorités locales. Durant cette première phase de développement, les projets d'investissements sont limités en taille et se résument à quelques dizaines, voire centaines de résidences. On peut ainsi interpréter l'arrivée des petits entrepreneurs comme une étape préalable à un développement de plus grande envergure.

Dans la seconde phase du boom (2006-2008), la consolidation des réseaux institutionnels et professionnels internationaux précipite l'arrivée de grandes multinationales immobilières et touristiques bien intégrées dans les circuits financiers internationaux, et pour la plupart originaires d'Europe ou des métropoles du sud-est brésilien ${ }^{3}$. Il s'agit désormais de projets de plus grande taille. Selon le Secrétariat du tourisme du RN, les 70 projets initiés en 2005 représentent un total de 27250 lits, soit $60 \%$ de la capacité 
existante à l'époque dans tout l'État (39252 lits) $)^{4}$. Dans le RN, à peine six projets concentrent à eux seuls près de 70000 résidences, une douzaine de terrains de golf et une dizaine de terrains de football.

À partir de 2008 (troisième phase), les marchés financiers internationaux s'effondrent, stoppant net les flux d'investissements européens. Les vols directs en provenance de l'Europe sont drastiquement réduits, ce qui renchérit le prix des billets et fait chuter le nombre de touristes européens et, par conséquent, de potentiels acheteurs. Les projets non encore concrétisés avortent et les projets en cours d'exécution sont redimensionnés pour s'adapter à la demande brésilienne, qui résiste un temps à la crise, jusqu'à l'essoufflement de l'économie nationale en 2012.

\section{Petits condominiums « entre amis »}

Bien que les mégaprojets focalisent fortement l'attention des observateurs et des médias, les petits projets des premières années du boom constituent la majorité des unités effectivement construites. Ces premiers projets sont surtout le fait de petits entrepreneurs extérieurs au milieu immobilier. Il s'agit le plus souvent d'entrepreneurs ayant connu le Nordeste à l'occasion d'un voyage ou par l'intermédiaire d'un proche. Leurs motivations sont partagées entre une volonté de reconversion personnelle, un certain «goût de l'aventure» et l'ambition d'en tirer une plus-value financière. L'expression «monter un condominium entre amis » revient régulièrement parmi les interviewés pour qualifier ces petits projets immobiliers. Jorge Mororo, un constructeur de Natal sollicité à plusieurs reprises par des investisseurs étrangers, décrit les premiers investisseurs comme «des groupes très fragmentés, des amis qui se réunissaient et formaient une société » (entretien, novembre 2009). Leur projet d'entreprise s'inscrit bien souvent dans une trajectoire de reconversion personnelle, à l'image de ce promoteur catalan actif sur Ponta Negra :

Je suis arrivé ici il y a dix ans, vers 2001. C'était dans l'idée de changer d'air, de diversifier un peu mes activités. Quand je suis arrivé, il n'y avait presque rien, tout cela n'existait pas. J'ai travaillé avec diverses entreprises immobilières en freelance pendant deux ou trois ans. En Espagne, j'avais une entreprise de serres agricoles, que j'ai vendue. Les gens en Espagne disent que la crise a commencé en 2007, mais pour moi ça a été en 2006. C'est là que j'ai vendu l'entreprise. À l'époque je faisais des allers-retours, je venais avec des clients, je faisais en sorte qu'à chaque fois que je vienne ça ne me coûte rien. C'est comme ça que je me suis fait mon trou, petit à petit...

-Mais avant tu ne travaillais pas dans l'immobilier alors?

-Un peu, mais surtout du «rustique», des maisons de campagne, des terrains agricoles.

-En plus de ton activité agricole?

-Oui, en plus... Ici j'ai commencé à travailler avec Real Construções, des Portugais qui travaillent à Pipa. J'avais un petit réseau de clientèle avec mon activité dans le milieu du rustique en Espagne. Donc entre les commissions et mon argent de côté, j'ai pu apporter un capital et on a crée l'entreprise ensemble. (Rafael, entrepreneur immobilier espagnol, entretien, mars 2010.)

On peut également citer le cas d'Álvaro, un ancien employé de l'entreprise hôtelière Marina d'Or, par ailleurs représentant consulaire espagnol pour le RN. Diplômé d'une prestigieuse école de commerce espagnole, il fréquente Natal depuis les années 1990 et connaît bien le milieu local des affaires. Avec trois amis catalans, il a lui-même «monté » 
un condominium, le Pipa Hills, une luxueuse résidence sécurisée composée d'une cinquantaine de villas dispersées au milieu d'un bois soigneusement préservé. La plupart des acheteurs ont été recrutés parmi ses réseaux de connaissances ou parmi des touristes espagnols ayant fait appel à lui en tant que consul pour résoudre des problèmes de passeports. Par exemple, Andrés et Marie-Carmen, un couple de quinquagénaires madrilènes (lui est enseignant en ophtalmologie, elle est entrepreneure), ont acheté une villa en 2006 sur les recommandations de collègues de travail, sans même l'avoir visitée au préalable, «à la confiance » :

Ça a été un coup de cœur vraiment. C'est vrai que c'était osé. Des collègues de travail avaient vu la zone et ça leur avait beaucoup plu. On est tous des ophtalmologues, donc c'était des connaissances... Ils ont rencontré Álvaro parce que l'un d'eux avait eu un problème avec son passeport. C'est là qu'ils ont su pour le projet du Pipa Hills. Ce sont eux qui nous ont donné l'idée. Et le fait d'avoir des interlocuteurs espagnols à Barcelone et ici, ça a beaucoup facilité les choses. (Andrés, résident secondaire espagnol, entretien, mars 2010.)

19 Lorsque les réseaux de connaissances ne suffisent pas, les promoteurs recrutent des agents immobiliers dans les régions émettrices en Europe. À Pipa, la plupart des agences comptent des salariés espagnols et britanniques, ou alors disposent de relais outreAtlantique, chargés de trouver des clients. À Alicante, j'avais rencontré l'un de ces relais, Pablo, un technicien en climatisation industrielle arrivé «un peu par hasard» au courtage immobilier. Il possède une page Internet au look désuet sur laquelle il annonce des offres de terrains et de résidences secondaires :

C'est le propriétaire de la Tour Casabalanca, quand il a essayé de nous vendre un appartement, c'est lui qui m'a introduit au sujet, d'abord pour que je vende du froid.

-Du froid?

-Oui l'idée, c'était que j'installe la climatisation sur des projets [...] Mais j'ai vu qu'il $\mathrm{y}$ avait des possibilités en termes de vente, il $\mathrm{y}$ avait des infrastructures très primaires.

-Mais tu avais déjà une expérience dans le négoce immobilier?

-Ce n'est pas que je me dédiais à la vente, mais j'avais des amis, des amis bien introduits. Et puis pour être originaire d'ici [Alicante], j'avais une certaine idée de comment ça marchait. L'expérience que j'avais acquise sur la Costa Blanca, j'essayais de l'implanter là-bas: l'idée c'était d'exporter le modèle de la Costa Blanca là-bas [au Brésil]. (Pablo, spécialiste en climatisation, agent immobilier, Espagnol, entretien, mai 2010.)

Que ce soit avec Rafael ou Pablo, tous deux intermédiaires immobiliers, on observe qu'en Espagne cette activité semble considérée comme une "seconde nature ». Même lorsqu'il ne s'agit pas de leur cœur de métier, ils sont déjà socialisés à la vente immobilière, soit à travers leurs expériences d'achat à titre personnel, soit par l'intermédiaire d'amis. En étant implantés dans des régions touristiques déjà connectées aux circuits internationaux de vente, ils pensent tirer profit des « externalités de réseau » de l'industrie immobilière espagnole et de la "culture de la brique» [cultura del ladrillo] acquise au cours de plusieurs décennies de croissance immobilière effrénée.

On retrouve le même mécanisme de vente en réseaux parmi les Norvégiens, aussi très présents à Pipa, où pas moins de quatre immeubles en copropriété ont été initiés par des Scandinaves. Le premier condominium a été lancé en 2002 par un petit groupe immobilier emmené par Kim, chef d'entreprise installé au Brésil dans les années 1990, "suite à une histoire d'amour ». La Colline des Norvégiens est une résidence fermée de onze maisons de vacances construites par des artisans locaux, dans un style proche des maisons des 
habitants « autochtones » [nativos]. Kim en a ensuite ouvert un second en 2008, Pipa Park, un immeuble plus moderne de 27 appartements, avec piscine et bar à cocktails. Là encore, les ventes se font parmi les réseaux de connaissances des promoteurs. J'ai rencontré un résident secondaire, Odd, ingénieur à la retraite de 62 ans, qui n'est autre que l'oncle de Kim. Il affirme avoir acheté " parce que c'était Kim ». Lorsque j'évoque le nom des deux autres condominiums appartenant à des Norvégiens, Kim parvient parfaitement à situer l'origine géographique des clients sur la carte norvégienne - «ceux-là sont tous de Tromsø!»-, indiquant que chaque établissement recrute sa clientèle sur une base territoriale différente. La promotion immobilière n'est pas l'activité principale de Kim, il exerce toujours une activité professionnelle en Norvège et le condominium lui permet de financer les quelques mois qu'il passe à Pipa chaque année. Ses deux enfants, issus d'un premier mariage avec une Brésilienne, sont scolarisés en Norvège, mais l'accompagnent pendant les vacances d'hiver (d'été au Brésil). Bien intégré à Pipa, où il a vécu à plein temps pendant plusieurs années, il s'enorgueillit d'avoir créé un projet immobilier socialement et territorialement ancré, élaboré « de bas en haut, et non pas de haut en bas » (entretiens réalisés en août 2011).

\section{Investissements « par amour »}

Si l'achat d'une résidence secondaire va bien au-delà du tourisme, les situations d'«apesanteur sociale» (Despres, 2017) et les imaginaires que recèle l'expérience touristique influencent le processus d'acquisition d'un bien « immobilier-touristique ». Le "relâchement de l'autocontrôle " (Réau, 2005) induit par la rupture avec le quotidien conduit parfois à des comportements « liminaux» aussi parmi les résidents secondaires, comme le constate Álvaro, gérant d'un condominium habité par une majorité de villégiateurs européens :

Tu en as qui font n'importe quoi, qui ramènent des filles chez eux et qui se mettent dans des états pas possibles. Des pères de famille, des choses qu'ils ne feraient jamais dans leur pays. Ils perdent la notion des choses, ils ne se rendent pas compte qu'ici aussi les gens parlent... (Álvaro, gérant d'un condominium et entrepreneur immobilier, espagnol, entretien, mars 2010.)

Il faut aussi envisager les continuités entre l'expérience amoureuse vécue en situation touristique et l'expérience migratoire aboutissant à une relation affective de long terme et, le cas échéant, à l'achat d'une résidence commune du couple sur ce même lieu de vacances. En effet, tant pour les promoteurs amateurs que les acquéreurs de résidence secondaire, la décision d'investir dans l'immobilier de loisir est souvent associée à un projet conjugal. Comme j'ai pu l'observer en consultant les registres fonciers de Praia da Pipa, dans le Rio Grande do Norte, un grand nombre de projets étaient portés par des couples mixtes, composés le plus souvent d'un homme étranger (européen) et d'une femme brésilienne.

Pour un étranger installé au Brésil, le fait d'avoir un(e) conjoint(e) brésilien(ne) permet de contourner un certain nombre de barrières administratives. Sur un plan légal, les normes de création d'une société imposent qu'au moins un associé soit Brésilien ou titulaire d'un visa de résident permanent, ce qui peut s'avérer utile dans le cas d'une entreprise immobilière. L'union avec un Brésilien facilite également l'obtention d'un visa permanent ${ }^{5}$. Une simple « union stable» - définie comme une "relation affective entre deux personnes, de caractère durable et avec pour objectif de constituer famille» 
(Loi 9.278/1996) - vaut une reconnaissance officielle du lien, sans que soit nécessaire la réalisation d'un contrat de mariage ou d'une union civile. Si cette disposition légale permet à des étrangers d'obtenir un visa permanent (une pratique connue sous le terme de "visa d'amoureux» [visto de namoro]), elle est aussi une source de litiges judiciaires lors des séparations. Car en l'absence de déclaration contraire, il prévaut automatiquement entre les compagnons en " union stable » un régime de communauté partielle des biens, ce qui signifie que les biens mobiliers et immobiliers acquis par l'un ou l'autre partenaire pendant la durée de la relation sont considérés comme étant le fruit d'une collaboration et appartiennent par conséquent à parts égales aux deux individus. Pour Kim, le Norvégien par deux fois divorcé, le droit matrimonial brésilien est jugé défavorable aux étrangers :

$\mathrm{Au}$ Brésil, il y a deux types de droits qui sont très développés: le droit des travailleurs et le droit des femmes. Si tu as un problème en justice avec un de tes travailleurs ou avec ta femme, c'est souvent en leur faveur. Autant dire que si tu as un problème avec une femme salariée, tu es mal parti! (Kim, investisseur immobilier et gérant de condominium, Norvégien, entretien, août 2010.)

En observant le déroulement des projets d'investissements réalisés par des hommes étrangers en couple avec des Brésiliennes, on comprend que même lorsque c'est l'homme qui apporte la majeure partie du capital, la femme joue très souvent un rôle de «culture broker » (Smith, 2001) dans le projet. Outre le fait que les femmes prêtent leur nom lors de la création de l'entreprise, leurs compétences linguistiques et sociales, leurs relations amicales ou familiales, sont souvent mises au service du projet immobilier pour aider à négocier avec l'administration locale ou avec les différents partenaires du projet (salariés, constructeurs, etc.), pour traduire certains documents, pour sensibiliser leur partenaire étranger aux subtilités du jeitinho brasileiro, l'art de l'arrangement «à la brésilienne ». Dans des administrations aussi bureaucratiques que les cartórios $^{6}$ (agences de services notariaux et de registres), dans les organes environnementaux (Loloum, 2016) ou dans les administrations communales, le fait de compter sur l'appui de personnes qui connaissent bien ces institutions peut s'avérer décisif pour accélérer les procédures.

Mettre en avant un(e) partenaire brésilien(ne) est aussi un moyen de s'éviter les discriminations en demi-teinte liées au statut d'étranger. Il est ainsi fréquent que le partenaire national mette à profit ses connaissances locales pour jouer un rôle de courtier immobilier au profit du projet d'investissement, voire d'intermédiaire politique ${ }^{7}$. À cela s'ajoute le «travail déguisé » que les femmes pourront apporter dans les établissements de leur compagnon, un phénomène largement répandu dans l'activité hôtelière réputée pour faire des « tâches féminines » une marchandise (Guerrier, 1986 ; Sinclair, 1997).

On aurait tort pour autant de croire que la femme est systématiquement instrumentalisée ou située dans une position de subordination par rapport à l'homme étranger. À l'encontre de l'imaginaire victimaire habituellement véhiculé à travers le thème du "tourisme sexuel", les informations recueillies témoignent au contraire d'une forte « capacité d'agence » (ou agentivité) des femmes brésiliennes (Roux, 2011). Le partenaire national effectuant ce travail de « courtage culturel » peut aussi utiliser ces compétences comme levier d'ascension sociale ou comme un élément de négociation dans le partage des ressources de l'entreprise conjugale.

Par ailleurs, l'enquête menée par Girona et ses collègues (2012) au sujet des «migrations par amour » entre Espagnols et Brésiliennes invite à questionner les représentations communes plaçant systématiquement le conjoint brésilien dans une position 
socioéconomique inférieure à celle du conjoint européen. De fait, leur enquête montre qu'il est courant que les femmes mariées à des étrangers présentent un niveau de formation équivalent ou supérieur à leur partenaire européen, en particulier dans le contexte de années 2008-2012 où l'Europe plongeait dans une longue crise économique alors que le Brésil semblait y résister8. En dépit de l'apparente « exogamie radicale » que constitue la formation d'un couple mixte, on retrouve bien souvent une forte « homogamie sociale » entre les partenaires, ou du moins une homologie des trajectoires (Loloum, 2017). C'est parfois l'incapacité des hommes européens à trouver une femme dans leur entourage (Girona, 2007) ou une inadaptation aux nouveaux modes sociaux de relations amoureuses dans leur pays (Bourdieu, 2002) qui les poussent à chercher une compagne étrangère. Il n'y a donc pas de corrélation automatique entre différence nationale, division de sexe et inégalité sociale. Il faut notamment considérer les modes de rencontre, les sens de circulation et les contextes migratoires. Contrairement aux configurations conjugales étudiées par Girona et ses collègues (2012), où les hommes espagnols sont toujours présentés comme des "touristes amoureux" ou des "importateurs d'amour", et où les Brésiliennes sont toujours présentées comme des «migrantes par amour», ici ce sont les hommes étrangers qui s'installent au Brésil comme «migrants» et les femmes brésiliennes qui sont dans une position d'« importatrices». On peut dès lors distinguer les couples formés à l'étranger, qui laissent supposer une origine sociale aisée de la femme ayant acquis un capital " mobilitaire » avant la rencontre ${ }^{9}$, des couples formés au Brésil où l'inégalité sociale (et d'âge) entre l'homme étranger et la femme brésilienne peut s'avérer importante ${ }^{10}$. Ces cas sont évidemment des idéaux types, et l'on pourrait sans doute multiplier les cas singuliers d'unions mixtes, à l'instar du Norvégien Kim qui a rencontré sa femme, une Carioca issue d'un milieu aisé, à l'occasion d'un voyage à Rio. Néanmoins, il est clair que les configurations conjugales jouent un rôle aussi déterminant que méconnu dans la menée des projets immobiliers, plus particulièrement en contexte international où la mixité du couple permet de contourner certaines difficultés d'installation, tout en complexifiant la situation sur le plan affectif.

Pour les hommes interrogés, investir dans l'hôtellerie ou l'immobilier est souvent perçu comme le signe d'une migration positive et volontariste, une façon de « réaliser un vieux rêve ». Pourtant, créer une entreprise est bien souvent l'une des rares façons pour eux de se constituer une situation professionnelle stable dans un pays où ils maîtrisent mal la langue et où ils gagneraient sans doute beaucoup moins en tant que salariés que ce qu'ils gagnaient en Europe. C'est du moins ce que laisse entendre Kim, le promoteur norvégien cité plus haut: "Ouvrir une auberge, c'est facile. Il y en a beaucoup qui font ça. Tu achètes une maison, tu loues quelques chambres et c'est parti. Moi je me suis juré que c'est quelque chose que je ne referais jamais. Tu deviens prisonnier de ton auberge.» Aussi, de la même manière que les migrations des femmes brésiliennes en Europe sont souvent source d'isolement social du fait de leurs difficultés à s'insérer dans le marché de l'emploi et de leur dépendance vis-à-vis des réseaux sociaux familiaux du conjoint (Girona, 2007), il n'est pas rare qu'une fois installés au Brésil les hommes étrangers se retrouvent en situation d'isolement et de dépendance vis-à-vis de leur compagne et de leur entourage. C'est par exemple ce que laisse entendre ce restaurateur suisse-allemand installé à Natal, qui a abandonné son premier restaurant à Salvador de Bahia en raison d'un désaccord avec son ex-femme et sa famille : « La famille était tout le temps chez nous et dans le restaurant, ça n'allait pas. Elle voulait travailler avec ses frères qui ne 
connaissaient rien à la restauration. Il y avait des choses qui disparaissaient... Bref, je lui ai dit 'garde le restaurant', et je suis parti. » (Peter, entretien, août 2011.)

Dans son étude au sujet de femmes européennes expatriées en Tanzanie, Despres (2017) soutient que le secteur touristique offre de bonnes perspectives d'emploi aux Occidentaux, qui peuvent «truster [...] les postes les plus qualifiés, nécessitant la maitrise d'une ou plusieurs langues internationales». Elle ajoute qu'elles bénéficient d'un «meilleur niveau de vie » et de " conditions de travail [leur] permettant d'économiser » ( ibid. : 74). Dans le cas du Nordeste brésilien, il s'avère bien difficile d'évaluer les gains en termes de «niveau de vie » ou de "qualité de vie », qui sont des notions subjectives. Si le secteur touristique offre en effet des débouchés intéressants aux étrangers, ceux-ci n'en restent pas moins en concurrence avec des travailleurs brésiliens originaires des grandes métropoles, tout aussi qualifiés et bien souvent polyglottes. Et si les salaires de cadres du tourisme sont relativement élevés par rapport au niveau salarial local, le coût de la vie est généralement plus élevé pour les étrangers qui veulent garder la possibilité de retourner en Europe, souvent plusieurs fois par an. Vivre au Brésil suppose par ailleurs de nombreux "coûts cachés " pour un citoyen européen habitué à un niveau élevé de services publics : «La vie est chère en réalité, surtout si tu as des enfants. Une bonne école ici te coûte 2000 réaux par mois. À cela il faut ajouter le coût de l'assurance santé, les problèmes à répétition avec l'administration qui peuvent te prendre un temps fou. » (Marc, hôtelier français, père de deux enfants, entretien, décembre 2015.) Le terme générique de "coût Brésil» [Custo Brasil] est souvent employé dans le jargon des entrepreneurs brésiliens pour décrire l'ensemble des difficultés structurelles qui peuvent affecter un investissement, qu'il s'agisse des coûts associés à la corruption, à l'insécurité juridique, aux délais administratifs, à la bureaucratie, aux difficultés d'accès à la maind'œuvre qualifiée, etc. Plus généralement, ces remarques renvoient aux difficultés pour certains étrangers récemment installés à s'adapter aux codes culturels des relations sociales, dont l'apparence de "cordialité » - accentuée par les imaginaires touristiques dissimule bien souvent la violence et la complexité des rapports sociaux de classe, de « race » et de sexe (DaMatta, 1991). L'expression consacrée par le musicien Antonio Carlos Jobim, «le Brésil n'est pas fait pour les débutants » [0 Brasil não é para principiantes], plusieurs fois entendue lors de mon enquête de terrain, évoque les malentendus que peut causer cette complexité des relations sociales pour l'étranger non initié. Dans ce contexte, la mixité du couple peut aussi bien constituer une source de malentendus et de conflits liés à l'interculturalité qu'une ressource pour contourner les difficultés de l'installation et les épreuves de la migration.

\section{Conclusion}

31 Au-delà des représentations communes associées à la mixité conjugale et aux migrations par amour, qui tendent à projeter des imaginaires postcoloniaux sur le couple, la présente étude - mise en regard avec la littérature existante sur l'expatriation amoureuse - tend à montrer la variété des configurations socio-affectives des couples transnationaux. Elle invite à considérer les conditions sociales de l'expatriation (les positions objectives des conjoints, leurs ressources respectives, leur disponibilité) en prenant en compte les conditions de départ mais aussi les contextes d'arrivée. Les chances de succès d'une expatriation varient en effet selon que l'on est une femme ou un homme, que l'on dispose d'un capital (financier) de réserve ou non, de compétences linguistiques ou d'une 
connaissance préalable du pays, que l'on est jeune ou moins jeune, que rien (ou presque) ne retient la personne qui part ou que, au contraire, l'expatriation implique d'abdiquer de nombreux avantages matériels, affectifs ou professionnels dans le pays d'origine. En d'autres termes, les chances de succès de la migration dépendent des dispositions et des disponibilités au départ. J'ai montré que la décision d'expatriation coïncidait souvent avec des moments de rupture dans la trajectoire professionnelle (réorientation, départ en retraite), sociale ou amoureuse (rupture, remariage, "crise de la quarantaine»). De même, les chances varient en fonction de la région d'expatriation, des perspectives professionnelles à l'arrivée (comparativement aux pays d'origine) et des différents coûts et avantages liés à l'installation. Le caractère concurrentiel du marché du travail, le coût de la vie, le niveau de salaire (comparativement au niveau de salaire dans le pays d'origine), le taux de change, le coût des transports aériens (pour occasionnellement retourner " au pays ») sont autant de facteurs qui entrent en ligne de compte.

À l'encontre des visions tantôt instrumentales tantôt romantiques des couples transnationaux, l'analyse des situations conjugales révèle l'ambiguïté de l'expatriation qui ne se fait jamais complètement - aveuglement - «par amour ", ni jamais totalement "par intérêt». Dans le contexte turbulent des situations migratoires et face à l'expérience de l'investissement immobilier, la mixité conjugale peut s'avérer une opportunité, permettant la transmission complémentaire de compétences et de ressources entre les conjoints, autant qu'une épreuve, du fait des inégalités socioculturelles et des relations de dépendance pouvant s'immiscer entre "l'importateur d'amour » et l'importé, peu importe leur sexe.

33 À la différence de nombreuses études portant sur les expériences amoureuses et sexuelles en contexte touristique, l'étude des investissements immobiliers comme point de départ des intimités transnationales permet de porter l'attention sur ces relations conjugales qui survivent à la parenthèse des vacances. Bien loin de la situation d'apesanteur sociale et "d'évasion temporaire» (Réau, 2005) que représente le voyage pour le couple, l'investissement «im-mobilier» est synonyme d'ancrage et d'engagement sur le long terme. Il ouvre dès lors sur un nouveau rapport au couple, mais aussi au pays et à l'environnement local. Là où le simple «touriste» pouvait aisément s'abstraire des difficultés - toujours temporaires - liées à sa position d'«outsider ", d'étranger, de " gringo », le conjoint qui s'installe doit composer avec cette identité assignée, dans des situations difficiles de négociation avec des administrations, des salariés, des collaborateurs, des voisins, une belle-famille, etc.

Bien loin des conceptions néoclassiques de l'économie, les différents cas présentés dans cet article rappellent l'encastrement des décisions économiques dans les sociabilités intimes (familiales, amicales et amoureuses). Cette dimension est d'autant plus saillante en contexte international et interculturel, où les évidences du genre, du couple et de la famille sont constamment remises en question. L'achat d'un bien immobilier, le démarrage d'une entreprise hôtelière, l'ouverture d'un restaurant sont toujours des épreuves personnelles. Ce sont aussi bien souvent des épreuves pour le couple, un engagement réciproque qui projette deux personnes dans un avenir commun. Au risque financier de l'investissement immobilier s'ajoutent l'insécurité culturelle et linguistique de vivre dans un nouvel environnement national, l'incertitude de cohabiter avec la bellefamille, de découvrir une nouvelle facette de son ou sa partenaire, de quitter son pays, ses proches. 
La dimension touristique des lieux investis peut à son tour renforcer le décalage entre, d'un côté, les attentes d'une vie plus «libre ", l'imaginaire idyllique de la destination balnéaire et, de l'autre, la réalité ordinaire de vivre à l'année dans un lieu de vacances, captif de son établissement et d'une communauté locale pour laquelle on reste assigné derrière l'étiquette de l'étranger. Le contexte instable et spéculatif des années 2000 a sans doute aussi joué sur les «esprits animaux » des investisseurs. Face aux incertitudes du marché touristique et aux complexités administratives du secteur immobilier, le capital social (le couple, la famille, les amis) constitue bien souvent une aide précieuse. L'anthropologie économique a déjà montré que la personnalisation des relations économiques est d'autant plus forte que le marché est opaque (Dufy et Weber, 2007 : 42-43). Le conjoint national (ou son entourage) peut ainsi jouer un rôle de «culture broker » (Smith, 2001) pour par exemple trouver un associé de confiance, gérer la négociation avec les administrations locales et les partenaires, faire jouer son réseau de relations afin de faire avancer un dossier ou contourner une difficulté administrative, assurer la maintenance de l'établissement en cas d'absence (les voyages à l'étranger sont logiquement plus fréquents dans le cas d'un couple mixte), trouver de la main-d'œuvre, et ainsi de suite. Ces "transactions intimes" (Zelizer, 2005) peuvent être une source d'enrichissement pour le couple et son entreprise, aussi bien qu'un facteur de dépendance (et donc de vulnérabilité) pour le conjoint expatrié. C'est en ce sens que dans les investissements immobiliers internationaux " par amour ", l'intime peut être à la fois une ressource et une épreuve.

\section{BIBLIOGRAPHIE}

Akerlof, George A. et Robert J. Shiller, 2009, Animal Spirits: How Human Psychology Drives the Economy, and Why It Matters for Global Capitalism, Princeton, Princeton University Press.

Alber, Jean-Luc, Laurence Ossipow, Valérie Outemsabet et Barbara Waldis, 2012, Mariages tous azimuts. Approche pluridisciplinaire des couples binationaux, Fribourg, Éditions universitaires de Fribourg.

Bessière, Céline, 2010, De génération en génération. Arrangements de famille dans les entreprises viticoles de Cognac, Paris, Raisons d'agir.

Bottero, Marion, 2015, Tourisme sexuel et relations conjugales en Thailande et en Malaisie, Paris, L'Harmattan.

Bourdieu, Pierre, 2002, Le bal des célibataires : crise de la société paysanne en Béarn, Paris, Seuil. Cantalice, Tiago, 2016, Dando um Banho de Carinho!: Os Caça-Gringas e as Interações Afetivo-Sexuais em Contextos de Viagem Turística (Pipa-Rn) [Pour un bain d'affection! Beach boys et interactions affectives-sexuelles en contexte touristique (Pipa, RN)], Jundiaí, Paco Editorial.

Constable, Nicole, 2003, Romance on a Global Stage. Pen Pals, Virtual Ethnography and "Mail Order" Marriages, Berkeley, University of California Press. 
Cueva, Carlos, R. Edward Roberts, Tom Spencer, Nisha Rani, Michelle Tempest, Philippe N. Tobler, Joe Herbert et Aldo Rustichini, 2015, Cortisol and Testosterone Increase Financial Risk Taking and May Destabilize Markets, Scientific Reports, Nature Publishing Group, <https://www.nature.com/ articles/srep1120>, consulté le 1 mai 2018.

DaMatta, Roberto, 1991, Carnivals, Rogues, and Heroes: An Interpretation of the Brazilian Dilemma, Notre Dame, IN, University of Notre Dame Press.

David, Louis, 2012, The Social Construction of Real Estate Markets' Risk. The Case of a Financial Investments Cluster in Mexico City, Articulo - Journal of Urban Research, $\mathrm{n}^{\circ}$ 9, <http:// articulo.revues.org/2163>, consulté le 1 mai 2018.

Despres, Altaïr, 2017, « 'Venues pour les plages, restées pour les garçons' ? Du tourisme à l'expatriation amoureuse des femmes occidentales à Zanzibar ", Recherches familiales, vol. 1, $\mathrm{n}^{\circ} 14$, p. 67-78.

Dufy, Caroline et Florence Weber, 2007, L'ethnographie économique, Paris, La Découverte.

Ferreira, Angela Lúcia A., Alexsandro Ferreira Cardoso Silva et Maria A. Pontes Fonseca, 2009, « Dinâmica imobiliária, turismo e meio ambiente: novos cenários Urbanos » [Dynamique immobilière, tourisme et environnement : nouveaux scénarios urbains], dans Maria do Livramento Miranda Clementino et Zoraide Souza Pessoa (dir.), Natal, uma metrópole em formação [Natal, une métropole en formation], Natal, RN, Observatório das Metrópoles, Núcleo RMNatal, p. $115-142$.

Fundação Getúlio Vargas, Ministério do Turismo, Embratur, 2008, Mercado de Turistas Estrangeiros de Segunda Residência no Brasil. Relatorio Executivo [français], Brasília, Fundação Getulio Vargas.

Girona, Jordi Roca, 2007, « Migrantes por amor. La búsqueda y formación de parejas transnacionales » [Migrants par amour. La recherche et la formation de couples transnationaux], Revista de Antropología Iberoamericana, vol. 2, nº 3, p. 430-458.

Girona, Jordi Roca, Monserrat Masdeu et Yolanda Puerta, 2012, « Migraciones por amor: diversidad y complejidad de las migraciones de mujeres » [Migrations par amour : diversité et complexité des migrations de femmes], Papers: revista de sociologia, vol. 97, n 3, p. 685-707.

Guerrier, Yvonne, 1986, « Hotel Manager - An Unsuitable Job for a Woman? » The Service Industries Journal, vol. 6, $\mathrm{n}^{\circ}$ 2, p. 227-240.

Herold, Edward, Rafael Garcia et Tony Demoya, 2001, « Female Tourists and Beach Boys: Romance or Sex Tourism? », Annals of Tourism Research, vol. 4, n² 28, p. 978-997.

Kempadoo, Kamala, 2001, « Freelancers, Temporary Wives, and Beach-Boys: Researching Sex Work in the Caribbean », Feminist Review, $n^{\circ} 67$, printemps, p. 39-62.

L’Estoile, Benoît (de), 2014, « 'Money Is Good, but a Friend Is Better': Uncertainty, Orientation to the Future, and 'the Economy' ", Current Anthropology, vol. 55, nº S9, p. S62-S73.

Loloum, Tristan, 2015, Derrière la plage, les plantations. Ethnographie d'une "situation touristique " dans le Nordeste brésilien. Le cas de Tibau do Sul, RN, thèse de doctorat en anthropologie sociale et études du tourisme (cotutelle), Paris et Lausanne, École des hautes études en sciences sociales et Université de Lausanne.

Loloum, Tristan, 2016, « Le gouvernement de la nature - Tourisme et institutions environnementales dans le Nordeste brésilien ", L’Espace politique, $\mathrm{n}^{\circ} 28,<\mathrm{https}$ :// journals.openedition.org/espacepolitique/3716>, DOI : 10.4000/espacepolitique.3716. 
Loloum, Tristan, 2017, « Contacts par homologie ? La fabrique des affinités en situation touristique », EspacesTemps.net, Travaux, 1 novembre 2017, <https://www.espacestemps.net/ articles/contacts-par-homologie/>.

Loloum, Tristan et Antonio Aledo, 2018 [à paraître], « La fabrique d'un boom immobiliertouristique. Tourisme et spéculation dans le Nordeste brésilien (2000-2010) », Norois, $\mathrm{n}^{\circ} 247$, avriljuin.

Piscitelli, Adriana, 2012, « Migración, género y sexualidad. Brasileñas en los mercados del sexo y del casamiento en España » [Migration, genre et sexualité. Les Brésiliennes sur les marchés du sexe et du mariage en Espagne], Mora, v. 18, p. 1-15.

Piscitelli, Adriana, 2016, « Erotics, Love and Violence: European Women's Travels in the Northeast of Brazil ", Gender, Place and Culture - A Journal of Feminist Geography, vol. 23, $\mathrm{n}^{\circ} 2$, p. 274-287.

Réau, Bertrand, 2005, «Évasions temporaires : socialisations et relâchements des contrôles dans les villages de vacances familiaux », Espaces et Sociétés, vol. 1-2, n ${ }^{\text {os }} 120-121$, p. 123-139.

Roux, Sébastien, 2011, No money, no honey. Économies intimes du tourisme sexuel en Thälande, Paris, La Découverte.

Simoni, Valério, 2016, Tourism and Informal Encounters in Cuba, New York, Berghahn Books.

Sinclair, Thea, 1997, Gender, Work, and Tourism, Londres, Routledge.

Smith, Valene, 2001, « The Culture Brokers », dans Valene Smith et Maryann Brent (dir.), Hosts and Guests Revisited: Tourism Issues of the $21^{\text {st }}$ Century, Elmsford, Cognizant, p. 275-282.

Zelizer, Viviana, 2005, « Transactions intimes », Genèses, n 42, p. 121-144.

Zelizer, Viviana, 2007, The Purchase of Intimacy, Princeton, NJ, Princeton University Press.

\section{NOTES}

1. Les entretiens ont été menés dans le cadre de ma thèse de doctorat portant sur les transformations sociales et politiques liées au tourisme dans une ancienne région de plantations de canne à sucre (Loloum, 2015). Le corpus mobilisé pour cet article concerne des entretiens réalisés auprès de professionnels du secteur immobilier (Brésiliens et Européens), ainsi que des investisseurs et des résidents secondaires étrangers (Italiens, Portugais, Espagnols, Français). Si les entretiens ne portaient pas directement sur les trajectoires biographiques des enquêtés, ni sur les questions intimes (mais plutôt sur leur perception de l'environnement local et leur expérience d'installation), ces questions apparaissaient immanquablement dans tous les entretiens, ou en marge de ceux-ci, lorsque le microphone était coupé et qu'ils se sentaient libres de parler des aspects intimes de leur expérience. L'observation participante et ethnographique, c'est-à-dire la cohabitation longue avec des couples mixtes installés dans le Nordeste (je fais chaque année depuis 2009 un ou deux voyages par an dans la région de Natal), a également constitué une source précieuse d'informations sur les économies intimes de l'expatriation amoureuse.

2. Historiquement, le commerce du sexe à Ponta Negra s'est développé depuis l'installation d'une base aérienne américaine pendant la Seconde Guerre mondiale.

3. Originaires de São Paulo surtout, capitale économique et financière du pays.

4. «Turismo lidera investimentos no RN » [Le tourisme en tête des investissements dans le Rio Grande do Norte], Tribuna do Norte, 14 novembre 2005. 
5. Sans visa de résident, un étranger ne peut rester que six mois par an, trois mois renouvelables une fois pour le «visa touriste». Une autre façon d'obtenir un visa de résident est de justifier d'un investissement minimum de plus de 50000 euros, ce qui implique de toute façon de créer une société. Les retraités étrangers jouissent quant à eux d'un statut particulier : ils doivent justifier d'un revenu mensuel minimum de $6000 \mathrm{R} \$$, soit 2000 euros environ.

6. Les cartórios sont des agences privées bénéficiant d'une délégation de pouvoir public pour garantir l'authenticité, la sécurité et la publication des actes juridiques. Elles sont chargées de tous les actes notariés, mais aussi de nombreux services qui, en Europe, serait pris en charge par les communes: actes de naissance, registres d'état civil, registres de biens immobiliers, etc. Institutions hybrides aux yeux d'un étranger, les cartórios sont un élément incontournable de la bureaucratie brésilienne. Ils accompagnent les citoyens tout au long de la vie, renforçant le rapport de familiarité entre usagers et agents notariaux et, par contraste, le rapport d'extériorité avec les étrangers.

7. À l'image de ce restaurateur portugais qui n'a pas hésité pas à inscrire sa femme (Brésilienne) dans un parti politique local pour s'accorder les faveurs des administrations municipales.

8. Longtemps épargné par la crise économique mondiale de 2008 grâce à une politique de la demande et un soutien à la consommation, le Brésil a par après été rattrapé par la récession à la suite de l'essoufflement des politiques de relance et d'une crise politique marquée par des opérations judiciaires de grande ampleur (en particulier l'Opération Lava Jato) et la destitution de la présidente Dilma Roussef en 2014.

9. C'est le cas par exemple de Grace, une avocate de Recife ayant rencontré son ex-mari en Allemagne lors d'un voyage d'études.

10. Cas de Portugais mariés avec des femmes natives de Pipa, généralement plus jeunes.

\section{RÉSUMÉS}

Partant d'une enquête ethnographique menée entre 2009 et 2014, au lendemain de la crise économique de 2008, dans une région touristique brésilienne (la côte du Nordeste) en prise avec d'importants investissements immobiliers internationaux, cet article rend compte de l'expérience d'entrepreneurs et d'investisseurs européens dans la concrétisation de projets immobiliers et touristiques sur le continent latino-américain. Suivant une approche anthropologique soucieuse de replacer les comportements économiques dans leur contexte social et culturel, l'enquête montre comment les «migrations par amour ", les projets conjugaux, les rapports de genre, les désirs et désillusions amoureuses, ainsi que les relations amicales et familiales des acteurs immobiliers peuvent influencer leurs décisions économiques. L'érotisation des imaginaires touristiques et le caractère international des relations de couple mixte dans lesquelles beaucoup de ces acteurs étaient engagés ajoutent une complexité supplémentaire à l'activité immobilière stricto sensu. En s'appuyant sur le concept d' « économies intimes ", invitant à questionner les frontières entre sphère de marché et sphère intime, l'objectif de l'article est de comprendre le rôle joué par les "expatriations amoureuses" dans la constitution de l'offre immobilière de destinations touristiques internationales. 
INDEX

Mots-clés : tourisme, immobilier, migrations, expatriation amoureuse, couples mixtes, Brésil

\section{AUTEUR}

\section{TRISTAN LOLOUM}

Ingénieur de recherche, Centres d'études supérieures de la Renaissance, Université de Tours ; Chercheur associé, Département d'anthropologie, Université de Durham ;

tristanloloum@gmail.com 